\title{
Disorder by Site
}

National Cancer Institute

\section{Source}

National Cancer Institute. Disorder by Site. NCI Thesaurus. Code C27551.

A term that refers to the classification of a disorder according to the anatomic site that is involved in the pathologic process. 